Check for updates

Cite this: RSC Adv., 2017, 7, 40452

Received 17th July 2017

Accepted 11th August 2017

DOI: $10.1039 / c 7 r a 07863 c$

rsc.li/rsc-advances

\section{Preparation of silver/reduced graphene oxide coated polyester fabric for electromagnetic interference shielding}

\author{
Can Wang, ${ }^{a}$ Cheng Xiang, ${ }^{a}$ Lin Tan, ${ }^{a}$ Jianwu Lan, ${ }^{a}$ Linghui Peng, ${ }^{a}$ Shouxiang Jiang ${ }^{b}$ \\ and Ronghui Guo (iD *a
}

In this study, silver/reduced graphene oxide (Ag/RGO) coated polyester (PET) fabrics with dopamine as adhesive agent were prepared through an efficient chemical reduction method under microwave irradiation. The as-synthesized samples were characterized by X-ray photoelectron spectroscopy (XPS), $X$-ray diffraction (XRD) and scanning electron microscopy. Deposit weight, surface resistance and electromagnetic interference (EMI) shielding effectiveness (SE) of the Ag/RGO coated PET fabric were investigated. The dopamine modified PET fabric is covered with silver particles and reduced graphene oxide (RGO) sheets uniformly and densely. In addition, the Ag/RGO coated fabric possesses high electrical conductivity with low surface resistance of $0.678 \Omega \mathrm{sq}^{-1}$ and EMI SE of Ag/RGO coated fabric ranges from $58 \mathrm{~dB}$ to $65 \mathrm{~dB}$ in the range of $1-18 \mathrm{GHz} \mathrm{X}$-band. Adhesive strength between $\mathrm{Ag} / \mathrm{RGO}$ particles and PET fabric is significantly improved.

\section{Introduction}

Recently, electromagnetic interference has received considerable attention because of the rapid development of electronic devices, which results in great negative effects not only on sensitive electronic equipment but also on human health. Therefore, it is urgent to develop textile materials which can protect the human body from electromagnetic interference.

Generally, EMI shielding efficiency is the combination of the reflection from the material surface, the absorption of electromagnetic energy and the multiple internal reflections of electromagnetic radiation. ${ }^{1}$ Among them, reflection is the determining factor for all high frequency signal attenuation, while maximum reflection occurs with the highest conductivity materials. Thus, EMI shielding properties depend mainly on the electrical conductivity. Poor electrical conductivity results in static electricity and transparent to electromagnetic wave. In recent years, graphene has been widely used in various fields due to its high surface area, high electrical conductivity, excellent mechanical and thermal properties. ${ }^{2-5}$ To date, many research groups have reported the feasibility of graphene used in EMI shielding and high-efficiency microwave absorption. ${ }^{6-9}$ $\mathrm{RGO}$, which is the derivatives of graphene, has received a great attention for functional materials. However, there are oxygen-

${ }^{a}$ College of Light Industry, Textile and Food Engineering, Sichuan University, Chengdu 610065, Sichuan, China. E-mail: ronghuiguo214@126.com; Fax: +86 28 85405423; Tel: +862865405420

${ }^{b}$ Institute of Textiles and Clothing, The Hong Kong Polytechnic University, Hung Hom, Kowloon, Hong Kong, China containing functional groups on RGO because of the incomplete reduction, which causes defects and restricts electrical conductivity of RGO. Currently, introducing metals or metal oxides on RGO has been considered to be an effective method for enhancing electrical conductivity. Recently, silver particles deposited on RGO to enhance functional properties has attracted ever-growing interest, because silver possesses excellent electrical conductivity, inoxidizability and antibacterial property and the synergistic effect of combination of graphene and silver particles. ${ }^{\text {10,11 }}$

Electrically conductive textile has been considered as wearable EMI shielding devices on account of its light weight, flexibility, and comfort. PET fabric has been widely used in textile industry because of its good wear resistance, high fracture strength and low production cost. However, it possesses disadvantages such as poor electrical conductivity and antistatic behavior. Hence, it is necessary of improving the electrical conductivity of PET fabric. Until now, there are a large number of researches on imparting PET fabric with high conductivity. In general, conductive polymer, metals and carbon materials are usually coated on PET fabric for enhancing electrical conductivity. ${ }^{12-14}$ In recent years, Babaahmadi and Moazami reported the synthesis of $\mathrm{Ag} / \mathrm{RGO}$ composites on PET fabric for improvement of electrical conductivity through traditional chemical reduction method. ${ }^{15,16}$ However, traditional chemical reduction method is usually carried out through heat conduction, which results in uneven heating during reaction because heating mode is from outside to inside. Thus, reaction rate is slow and the heating is not uniform.

Nowadays, numerous methods have been developed for assisting synthesis of $\mathrm{Ag} / \mathrm{RGO}$ hybrids such as visible-light, 
microwave and ultrasonic irradiation. ${ }^{17-19}$ Among them, microwave irradiation has been recently used as an important technique for assisting coating of materials on textile because microwave irradiation can offer rapid and uniform volumetric heating, high reaction rates, short reaction time, high yield production and low cost..$^{\mathbf{2 0}, 21}$ In addition, microwave irradiation is helpful for diffusion of molecules and ion into fibers and their further reduction.

It is difficult that materials are immobilized on fabrics owing to poor adhesion of the coating on fabrics. Therefore, it is very necessary and desirable to improve durability of Ag/RGO coatings on fabrics. Recently, dopamine has been used to modify surface of various textile materials to improve adhesive strength between coatings and substrates. ${ }^{22-24}$ Dopamine is a kind of biomolecule containing catechol and amine functional groups, thus it is eco-friendly and flexible agent for surface coating of various materials. Dopamine can be spontaneously selfpolymerized on a variety of substrates after being oxidized in air, which provides a facile method to immobilize desired coatings onto surface. ${ }^{25}$ However, to the best of our knowledge, it has not been reported that $\mathrm{Ag} / \mathrm{RGO}$ are coated on textiles with dopamine as an adhesive agent under microwave irradiation.

In this study, Ag/RGO coated PET fabrics modified with dopamine were prepared through chemical reduction under microwave irradiation. In addition, influences of concentration of silver nitrate in chemical reduction solution on deposit weight, surface resistance and EMI SE of Ag/RGO coated PET fabrics were investigated. The coated PET fabrics were characterized by XPS, SEM and XRD. Deposition rate, surface resistance and EMI SE of the coated PET fabrics were evaluated. Additionally, adhesive strength between Ag/RGO coatings and PET fabric modified with dopamine was evaluated.

\section{Experimental}

\subsection{Materials}

Plain weave $100 \%$ PET fabric $\left(95 \mathrm{~g} \mathrm{~m}^{-2}\right)$ in white color was used. Multilayer GO powder (purity > 95\%) was purchased from Suzhou Tanfeng Graphene Tech. Co., Ltd. Silver nitrate, trisodium citrate, tris(hydroxymethyl)aminomethane (Tris) and dopamine were purchased from Chengdu KeLong Chemical Co., Ltd. All the chemicals were of analytical grade and used without further purification.

\subsection{Modification of the PET fabrics with dopamine}

Dopamine $(0.3 \mathrm{~g})$ was dissolved in Tris buffer solution $(100 \mathrm{~mL})$. $\mathrm{pH}$ value of the solution was regulated to 8.5. PET fabrics were cut into $5 \mathrm{~cm} \times 5 \mathrm{~cm}$ and subsequently cleaned in acetone, ethanol and deionized water under ultrasonic irradiation for $30 \mathrm{~min}$, respectively. The cleaned PET fabrics were immersed into the solution with dopamine at ambient temperature for $24 \mathrm{~h}$ and then rinsed in deionized water.

\subsection{Synthesis of GO coated PET fabrics}

Aqueous dispersion of GO $\left(1 \mathrm{~g} \mathrm{~L}^{-1}, 100 \mathrm{~mL}\right)$ was prepared and sonicated for $60 \mathrm{~min}$. The dopamine modified PET fabrics were dipped into the prepared dispersion and then exposed to microwave irradiation (domestic microwave oven, $700 \mathrm{~W}$ ) for 20 min and turned over every two minutes to ensure the uniform coating GO nanosheets on the fabrics. The GO coated fabrics were rinsed in deionized water and finally kept in an oven at $100{ }^{\circ} \mathrm{C}$ for $60 \mathrm{~min}$.

\subsection{Synthesis of $\mathbf{A g} / \mathbf{R G O}$ coated PET fabrics}

GO coated PET fabric was immersed into the solution of silver nitrate with different concentration of silver nitrate $\left(1-6 \mathrm{~g} \mathrm{~L}^{-1}\right.$, $100 \mathrm{~mL}$ ). Trisodium citrate was added into the reaction mixture and the mixture was subjected to microwave irradiation (domestic microwave oven, $700 \mathrm{~W}$ ) for $20 \mathrm{~min}$. The fabrics were turned over every two minutes to ensure uniform coating of $\mathrm{Ag} / \mathrm{RGO}$ on PET fabrics. Finally, the Ag/RGO coated fabrics were rinsed in deionized water and then dried at $100{ }^{\circ} \mathrm{C}$ for $120 \mathrm{~min}$. For comparison, the RGO and $\mathrm{Ag}$ coated PET fabrics were prepared according to the above procedure, respectively. In addition, Ag/RGO with $6 \mathrm{~g} \mathrm{~L}^{-1}$ silver nitrate in the solutions was also coated on PET fabric without pretreatment with dopamine according to the above procedures. Additionally, Ag/RGO coated PET fabrics modified with dopamine with different silver nitrate concentration from $1 \mathrm{~g} \mathrm{~L}^{-1}$ to $6 \mathrm{~g} \mathrm{~L}^{-1}$ were labelled as $\mathrm{Ag} / \mathrm{RGO}$ D1 to Ag/RGO-D6, while Ag/RGO coated PET fabrics modified with dopamine was labelled as Ag/RGO-D. Besides, Ag/RGO coated PET fabric with silver nitrate concentration of $6 \mathrm{~g} \mathrm{~L}^{-1}$ without pretreatment with dopamine was labelled as Ag/RGO-N.

\subsection{Characterization}

Chemical composition of dopamine, GO and Ag/RGO coated PET fabrics was determined by XPS (Kratos XSAM800 spectrometer) using an $\mathrm{Al} \mathrm{K} \alpha$ source $(14 \mathrm{kV}$ and $350 \mathrm{~W})$.

Surface morphologies of original fabric and dopamine, RGO, $\mathrm{Ag}$ and $\mathrm{Ag} / \mathrm{RGO}$ coated PET fabrics were observed by SEM (JSM$5900 \mathrm{LV}$ ) at an acceleration voltage of $5 \mathrm{kV}$ and $10 \mathrm{~mm}$ of working distance.

Crystal structures of original fabric, and RGO and Ag coated PET fabrics and Ag/RGO-D6 were detected by XRD (X'Pert Pro MPO) with a scan rate of $5^{\circ}$ per minute in the angular range of $10-90^{\circ}$. The radiation used was $\mathrm{Cu} \mathrm{K} \alpha$ with a wavelength of $1.5418 \AA$.

Surface resistances of original fabric, and dopamine, RGO, $\mathrm{Ag}$ and $\mathrm{Ag} / \mathrm{RGO}$ coated PET fabrics were tested using a fourpoint probes equipment (RTS-9). Each sample was measured at least five times and the average values were calculated for the results.

EMI SE of original fabric and dopamine, RGO, Ag and $\mathrm{Ag} / \mathrm{RGO}$ coated PET fabrics were evaluated on a vector network analyzer (Agilent E5063A ENA) in 1-18 GHZ X-band range. Each sample was cut into circle shape with a diameter of $110 \mathrm{~mm}$ for the tested. Total EMI shielding effectiveness (SE (total)) was used to evaluate ability of material to attenuate electromagnetic waves, which refers to the logarithm of the ratio of the incident wave $P_{\mathrm{I}}$ to the transmitted wave $P_{\mathrm{T}}$ as shown in the following equation. 


$$
\mathrm{SE}=10 \log \left(\frac{P_{\mathrm{I}}}{P_{\mathrm{T}}}\right)
$$

$\mathrm{Ag} / \mathrm{RGO}$ coated PET fabrics were washed in $0.37 \mathrm{wt} \%$ of detergent with 10 steel balls at $40{ }^{\circ} \mathrm{C}$ for $45 \mathrm{~min}$ in accordance with standard AATCC 61-2003. SEM, surface resistance and EMI SE of Ag/RGO coated PET fabrics after washing were evaluated.

\section{Results and discussion}

\subsection{Mechanism of Ag/RGO coating on PET fabrics modified with dopamine}

The mechanism that Ag/RGO coatings are coated on PET fabric modified with dopamine under microwave irradiation is illustrated in Scheme 1. Dopamine was used as adhesive agent to improve the strength between coatings and textiles because it is difficult for $\mathrm{Ag} / \mathrm{RGO}$ immobilized on the surface of polyester fibers. Thus, thin film of GO can be can be quickly formed on PET fibers modified with dopamine when the PET fabric is immersed into aqueous solution of GO. Moreover, GO can be easily diffused into the gap among PET yarns under microwave irradiation. GO coated on the fibers carry negative charges due to the large quantities of carboxyl $\left(-\mathrm{COO}^{-}\right)$and hydroxyl $\left(-\mathrm{OH}^{-}\right)$ functional groups. Therefore, silver ions with positive charges can be easily adsorbed on the surface of GO coated fibers due to strong electrostatic force. Subsequently, silver particles and RGO sheets are formed through simultaneous in situ reduction of $\mathrm{Ag}^{+}$and $\mathrm{GO}$ to $\mathrm{Ag}^{0}$ and RGO with trisodium citrate as a reducing agent under microwave irradiation, respectively. Reaction efficiency is enhanced with the help of microwave irradiation because the reaction system can be heated quickly and uniformly. Therefore, microwave irradiation is beneficial for uniform deposition of silver particles and RGO sheets on the PET fibers.

\subsection{XPS analysis}

XPS wide and narrow spectra of dopamine, GO and Ag/RGO coated PET fabrics are illustrated in Fig. 1, 2 and 3, respectively. Elements of $\mathrm{C}$ and $\mathrm{O}$ detected from the fabric modified with dopamine (Fig. 1a) are attributed to PET fabric, while $\mathrm{N}$ comes from dopamine. Narrow spectrum of $\mathrm{N} 1 \mathrm{~s}$ is shown in Fig. 1b. It can be fitted into a typical peak at $402.0 \mathrm{eV}$, which is attributed to amine $(-\mathrm{N}-\mathrm{H}){ }^{26}$ The result confirms that dopamine is successfully adsorbed on surface of PET fabric.

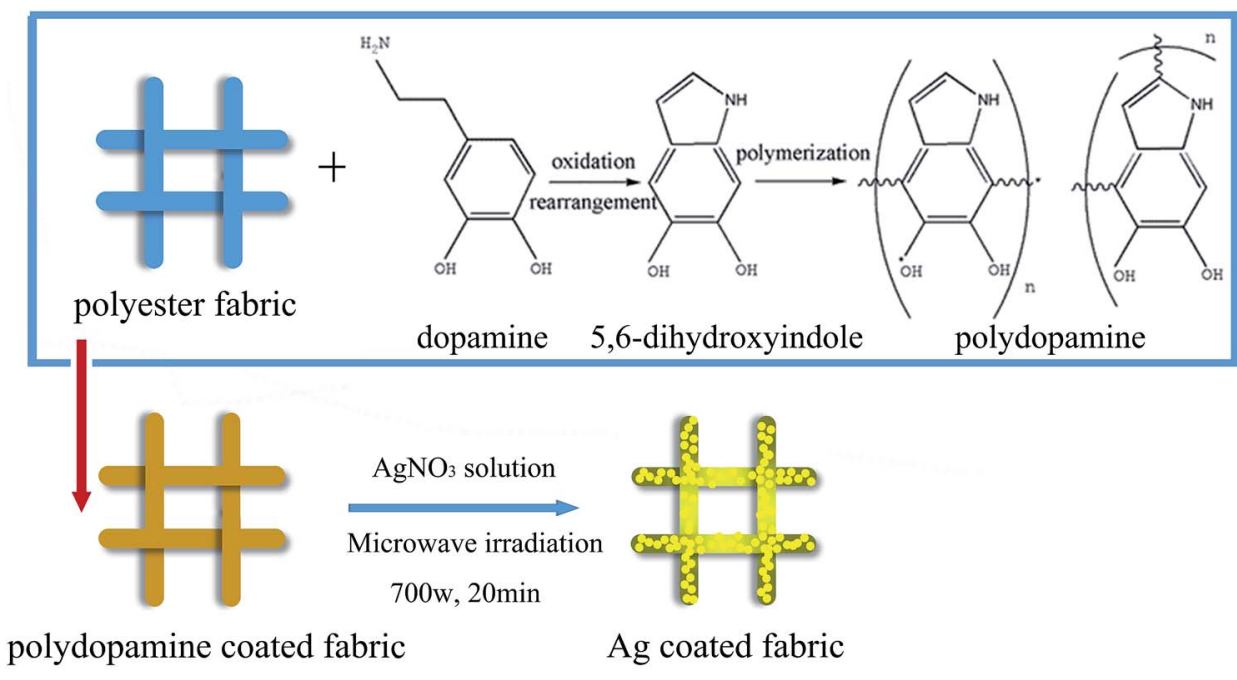

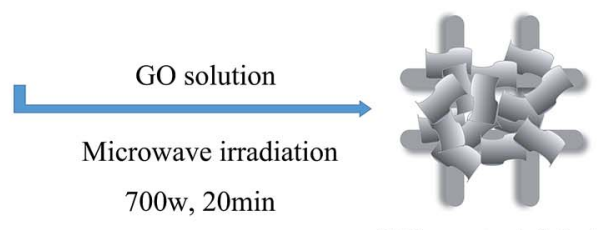

GO coated fabric

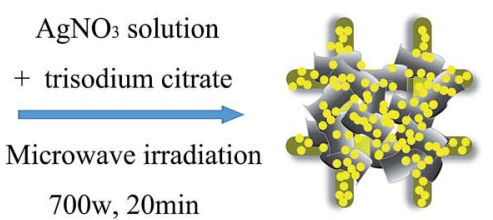

$\mathrm{Ag} / \mathrm{RGO}$ coated fabric

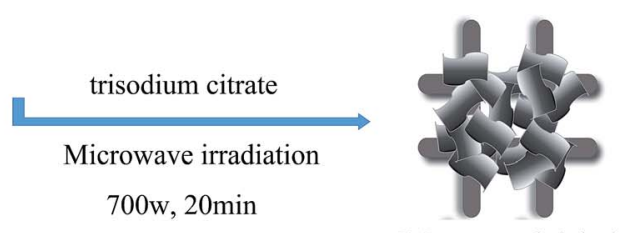

RGO coated fabric 

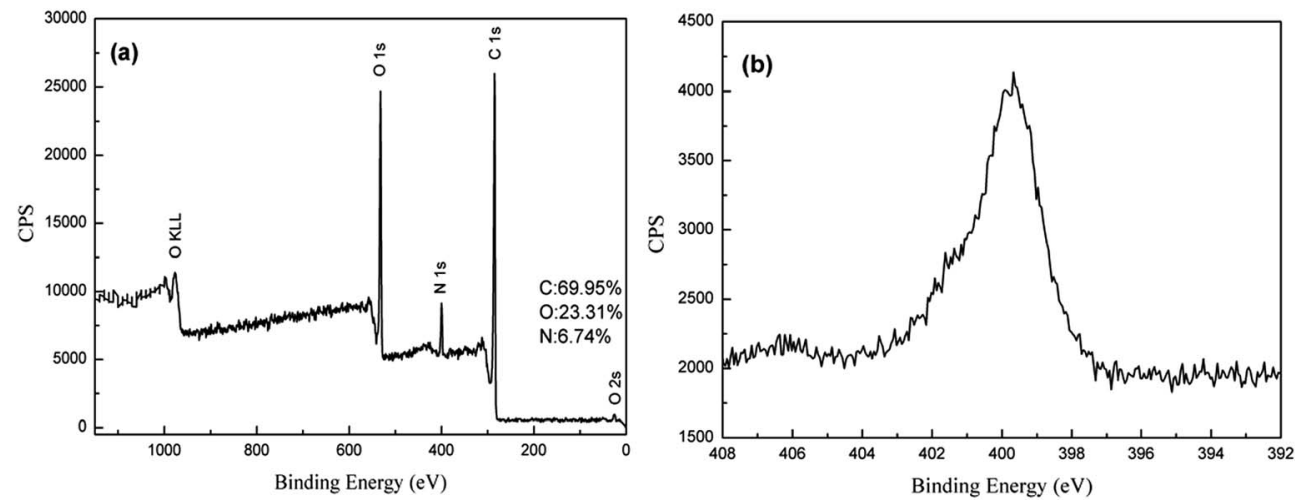

Fig. 1 (a) XPS wide and narrow spectra of (b) N 1s of PET fabric modified with dopamine.

XPS wide and narrow spectra of the GO coated PET fabric modified with dopamine are shown in Fig. 2. It is obvious that elements of $\mathrm{C}, \mathrm{O}$ and $\mathrm{N}$ are existed in the GO coated PET fabric modified with dopamine (Fig. 2a). It can be seen from Fig. 2c that $\mathrm{C}$ 1s spectrum of GO coated PET fabric can be deconvoluted into three peaks at $284.5 \mathrm{eV}, 286.7 \mathrm{eV}$ and $288.5 \mathrm{eV}$, which correspond to characteristics of bonds of $\mathrm{C}-\mathrm{C}$ (unoxidized graphite carbon skeleton), $\mathrm{C}-\mathrm{O}$ (hydroxyl and epoxide) and $\mathrm{C}=\mathrm{O}$ (carboxyl), respectively. The result is consistent with the reported literature. ${ }^{11,15,27}$

Three prominent elements of $\mathrm{Ag}, \mathrm{C}$ and $\mathrm{O}$ are existed in $\mathrm{Ag} / \mathrm{RGO}$ coated PET fabric as shown in Fig. 3. Elements of $\mathrm{C}$ and
$\mathrm{O}$ are ascribed to RGO and PET. In addition, $\mathrm{Ag} / \mathrm{RGO}$ coated PET fabric mainly consists of $\mathrm{Ag}$ and content of $\mathrm{Ag}$ is $89.48 \mathrm{wt} \%$ (Fig. 3a). The XPS narrow spectrum in the region $275-295 \mathrm{eV}$ is presented in Fig. 3b. It is obvious that peak intensity of C 1s on surface of Ag/RGO coated PET fabric at $286.7 \mathrm{eV}$ and $288.5 \mathrm{eV}$ are remarkably lower than that of GO coated fabric due to low content of $\mathrm{C}-\mathrm{O}$ and $\mathrm{C}=\mathrm{O}$ in $\mathrm{Ag} / \mathrm{RGO}$ coated PET fabric. The result confirms that GO is effectively reduced to RGO with trisodium citrate as a reducing agent under microwave irradiation. In addition, the peak of carbon species (C-C) at $284.1 \mathrm{eV}$ is strong. XPS narrow spectrum of $\mathrm{Ag} 3 \mathrm{~d}$ in the region of $360-380 \mathrm{eV}$ is presented in Fig. 3c. The peaks at $367.9 \mathrm{eV}$ and
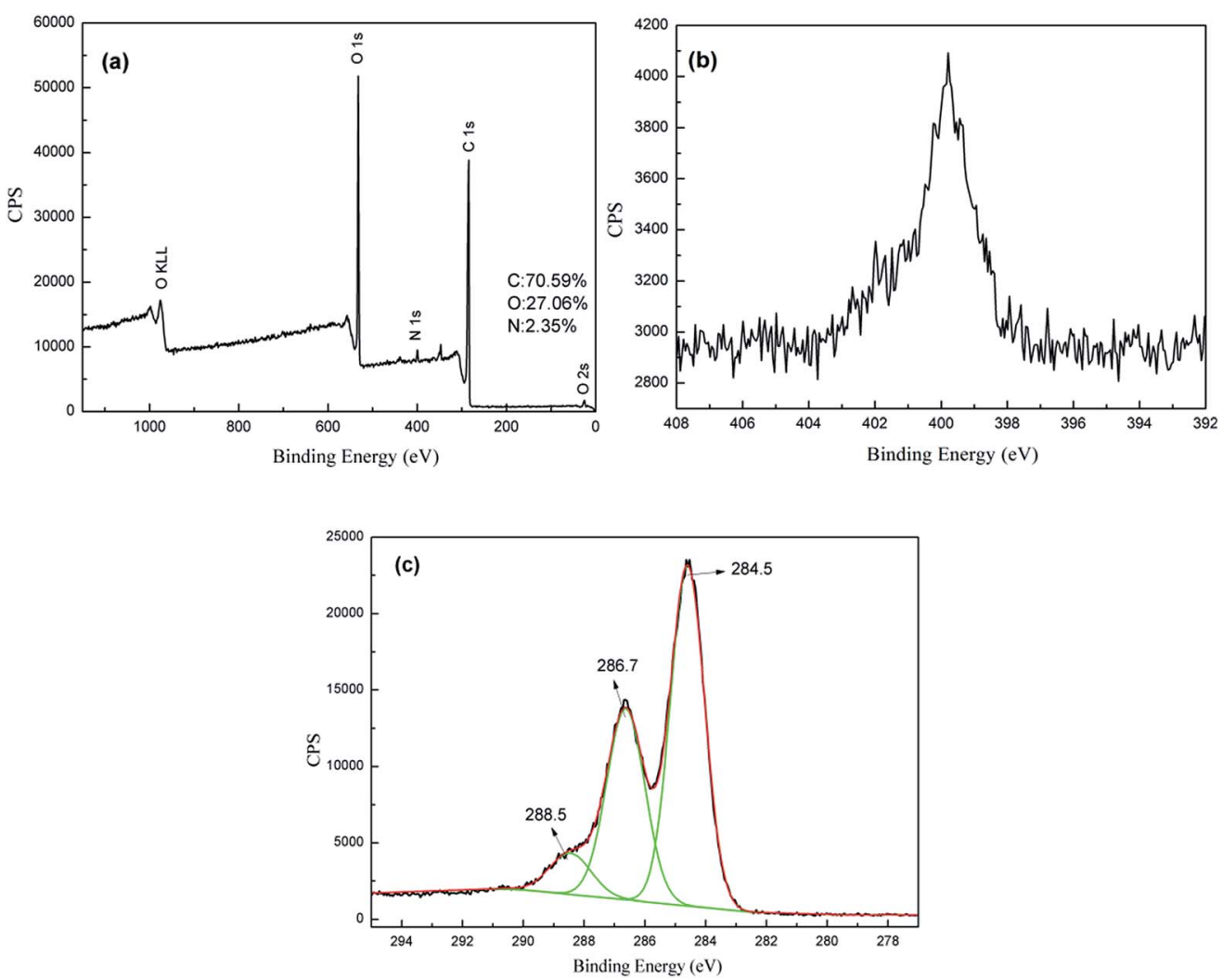

Fig. 2 (a) XPS wide and narrow spectra of (b) $N$ 1s and (c) $C$ 1s of GO coated PET fabric. 

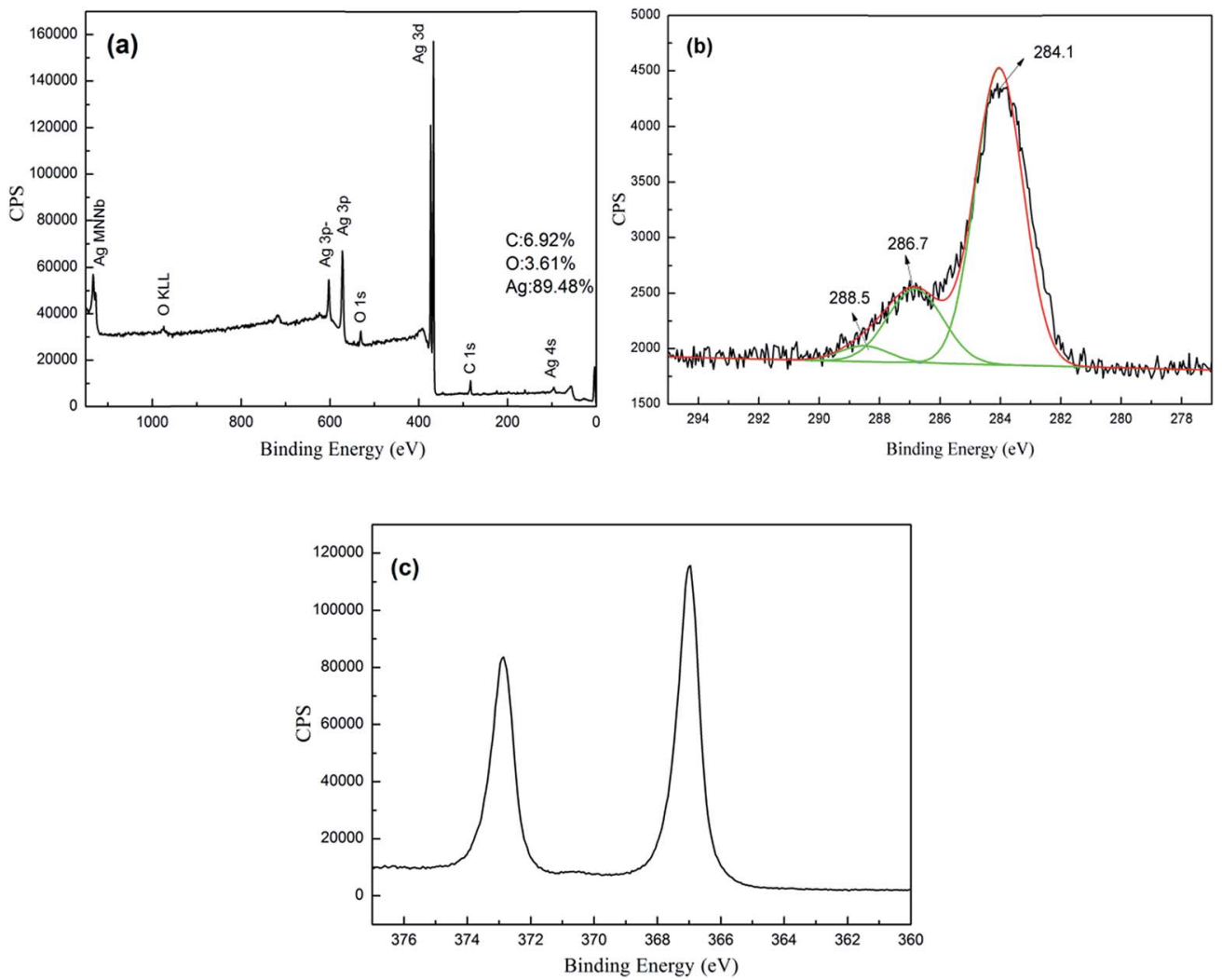

Fig. 3 (a) XPS wide and narrow spectra of (b) C 1s and (c) Ag 3d of Ag/RGO coated PET fabric.

$373.9 \mathrm{eV}$ correspond to $\mathrm{Ag} 3 \mathrm{~d} 5 / 2$ and $\mathrm{Ag} 3 \mathrm{~d} 3 / 2$ of metallic silver, respectively. The result indicates that silver ions are reduced to $\mathrm{Ag}^{0}$ under microwave irradiation. In conclusion, $\mathrm{Ag} / \mathrm{RGO}$ are successfully coated on the PET fabric.

\subsection{Surface morphology}

Fig. 4 shows surface morphologies of original fabric and dopamine, RGO and Ag coated PET fabrics and Ag/RGO-D6. It can be seen from Fig. $4 \mathrm{a}$ and $\mathrm{b}$ that PET fibers are cylindrical and surface is relatively smooth. The surface of PET fibers is covered with agglomerated polydopamine film (Fig. $4 \mathrm{c}$ and d) after dopamine modification. Fig. 4e and $\mathrm{f}$ show that RGO sheets are densely attached on the surface of PET fibers with slight winkles. The surface of Ag coated PET fibers is rough due to silver particles deposited on the fibers (Fig. $4 \mathrm{~g}$ and $\mathrm{h}$ ). Silver particles on the fibers modified with dopamine are irregularly round and the size ranges from $200 \mathrm{~nm}$ to $600 \mathrm{~nm}$. Surface morphology of Ag/RGO-D6 are presented in Fig. $4 \mathrm{i}$ and j. It is obvious that the silver particles are uniformly coated on the fibers and the particle size ranges from $300 \mathrm{~nm}$ to $1 \mu \mathrm{m}$. Silver particles of Ag/RGO-D6 is obviously bigger than that of $\mathrm{Ag}$ coated PET fabric. The phenomenon can be explained by the fact that $\mathrm{GO}$ film with oxygen atoms $\left(-\mathrm{COO}^{-}\right.$and $\left.-\mathrm{OH}^{-}\right)$shows strong electrostatic force to $\mathrm{Ag}^{+}$. The GO coated PET fabric can efficiently prompt silver deposition from the reaction solution, which would result in fast nucleation and growth of silver. The migration and aggregation of silver particles would produce thermodynamically stable particles with bigger sizes.
Fig. 5 presents surface morphologies of $\mathrm{Ag} / \mathrm{RGO}-\mathrm{N}$ and $\mathrm{Ag} /$ RGO-D6 after washing. It is obvious that a lot of Ag/RGO coatings are peeled off from the PET fibers without dopamine pretreatment after laundering according to standard method AATCC 61 (Fig. 5a and b). However, it can be seen from Fig. 5c and $\mathrm{d}$ that $\mathrm{Ag} / \mathrm{RGO}$ particles are still densely coated on the PET fibers modified with dopamine. Only few coatings are removed after laundering because of strong coordinate bonds and electrostatic adsorption between the coatings and fibers. Accordingly, adhesive strength between silver particles, RGO sheets and PET fibers is improved because coordinate bonds and electrostatic force between silver particles, RGO sheets and the polydopamine on the fabric are formed.

\subsection{Crystal structure}

X-ray diffraction patterns of RGO and $\mathrm{Ag}$ and $\mathrm{Ag} / \mathrm{RGO}$ coated PET fabrics are shown in Fig. 6. Weak peaks at $2 \theta$ of $17.2^{\circ}, 22.3^{\circ}$ and $25.5^{\circ}$ are assigned to PET fibers (Fig. 6a). The characteristic peak of RGO are obviously overlapped with peaks of PET. Five diffraction peaks of $\mathrm{Ag}$ and $\mathrm{Ag} / \mathrm{RGO}$ coated PET fabrics located at $38.1^{\circ}, 44.2^{\circ}, 64.3^{\circ}, 77.2^{\circ}$ and $81.5^{\circ}$ are assigned to (111), (200), (220), (311) and (222) reflection lines of silver, respectively (Fig. 6b and c). The XRD patterns identified by the PDF card reveals that $\mathrm{Ag}$ and $\mathrm{Ag} / \mathrm{RGO}$ coatings on PET fabrics exhibits face-centered cubic crystalline structure.

Based on the XRD results, crystal size of Ag deposits was determined from the broadening of the diffraction peak of (111) 


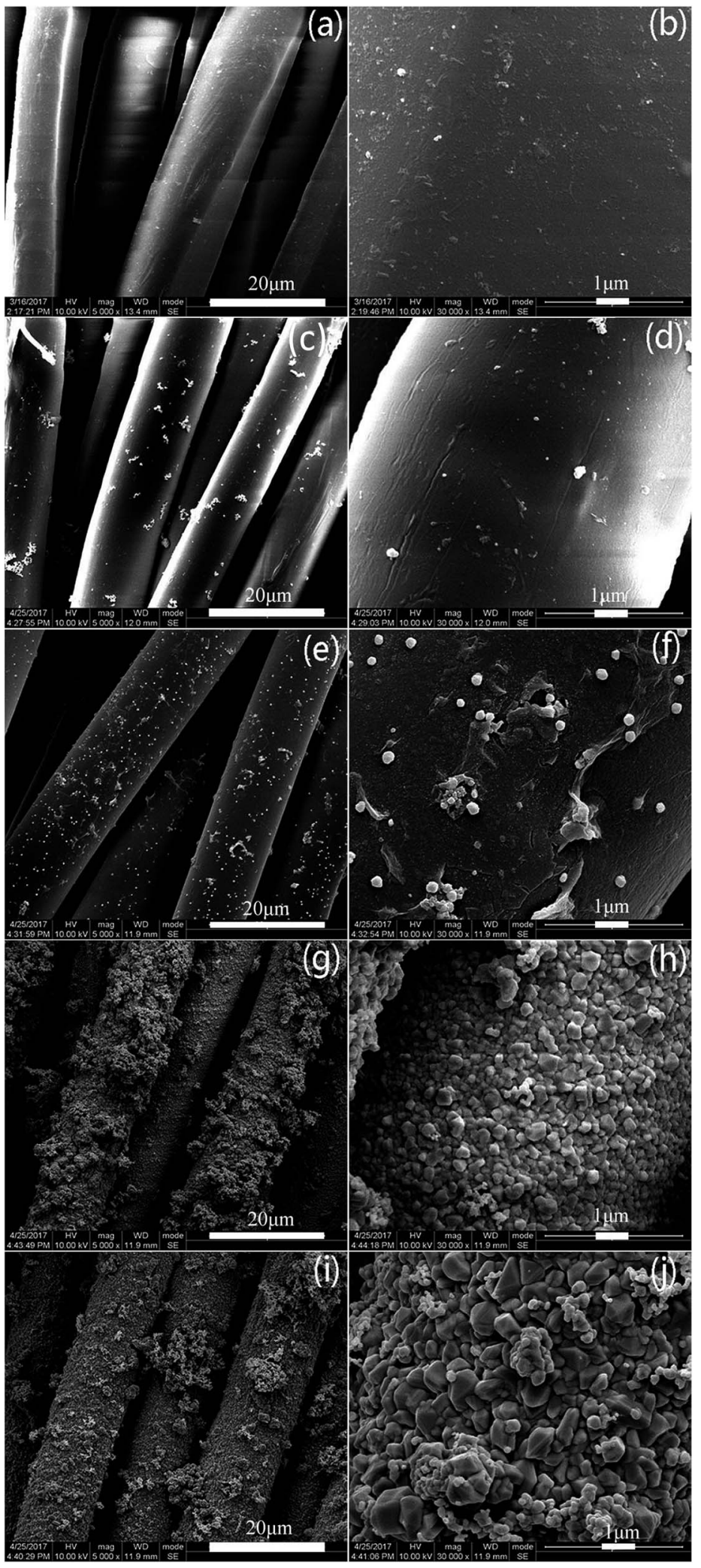

Fig. 4 SEM images of $(a, b)$ original PET fibers, $(c, d)$ dopamine, $(e, f) R G O$ and $(g, h)$ Ag coated PET fibers and $(i, j)$ Ag/RGO-D6. 


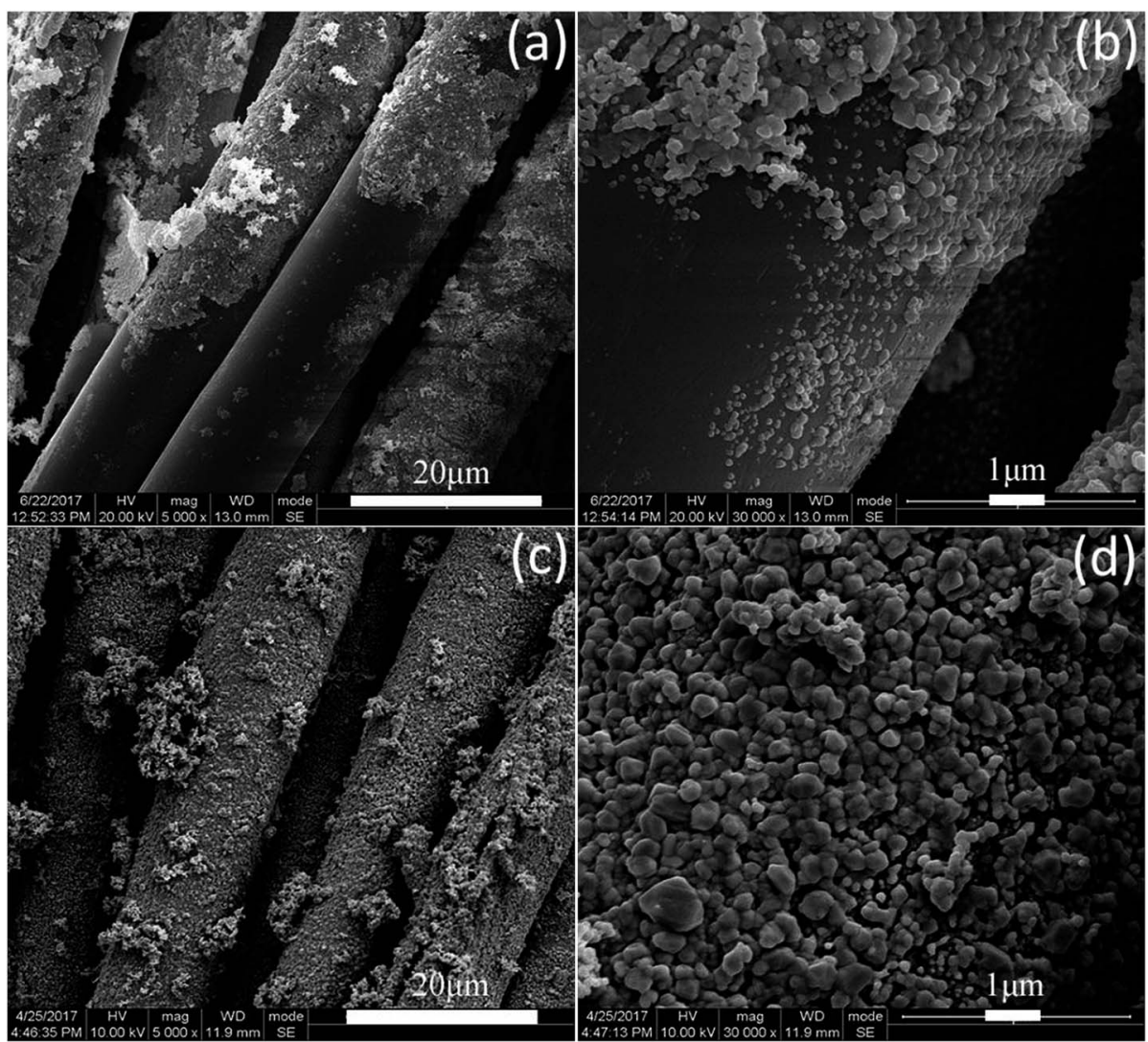

Fig. 5 SEM images of $(a, b)$ Ag/RGO-N and (c, d) Ag/RGO-D6 after washing.

by employing Scherrer formula as expressed by following equation.

$$
t=\frac{n \lambda}{B \cos \theta}
$$

where $t$ is the crystal size; $\lambda$ is the X-ray wavelength corresponding to $\mathrm{Cu} \mathrm{K} \alpha$ radiation $(0.154056 \mathrm{~nm}) ; \theta$ is the diffraction angle; $B$ is the full width half maximum (FWHM) of the diffraction peak at $2 \theta$ and $n$ is the Scherrer constant as 0.89 .

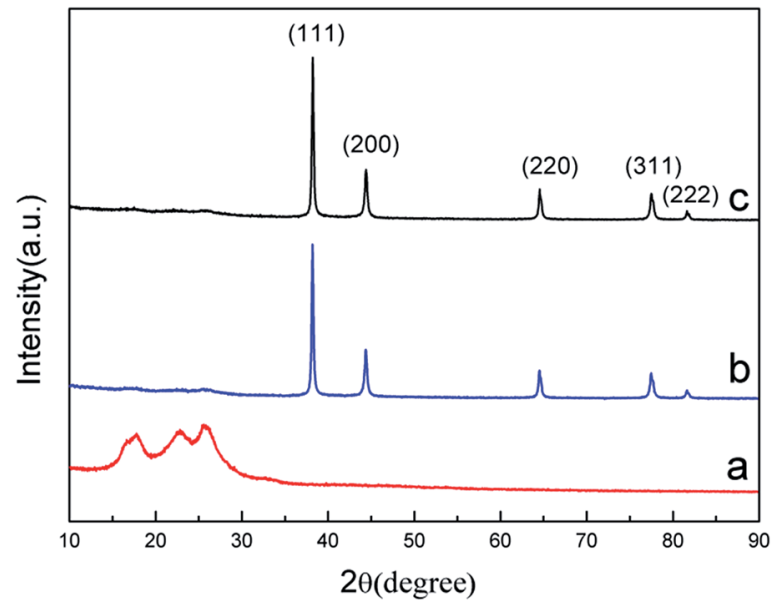

Fig. 6 XRD patterns of (a) RGO and (b) Ag coated PET fabrics, and (c) Ag/RGO-D6.
Average size of silver particles on the Ag coated PET fabric and $\mathrm{Ag} / \mathrm{RGO}-\mathrm{D6}$ are $29.89 \mathrm{~nm}$ and $30.83 \mathrm{~nm}$ with respect to $\mathrm{Ag}$ (111) peak, respectively.

\subsection{Deposit weight and surface resistance}

Surface resistance and deposit weight of Ag coated PET fabric and Ag/RGO-D are shown in Fig. 7. As is well known, the original and dopamine coated PET fabrics show no electrical

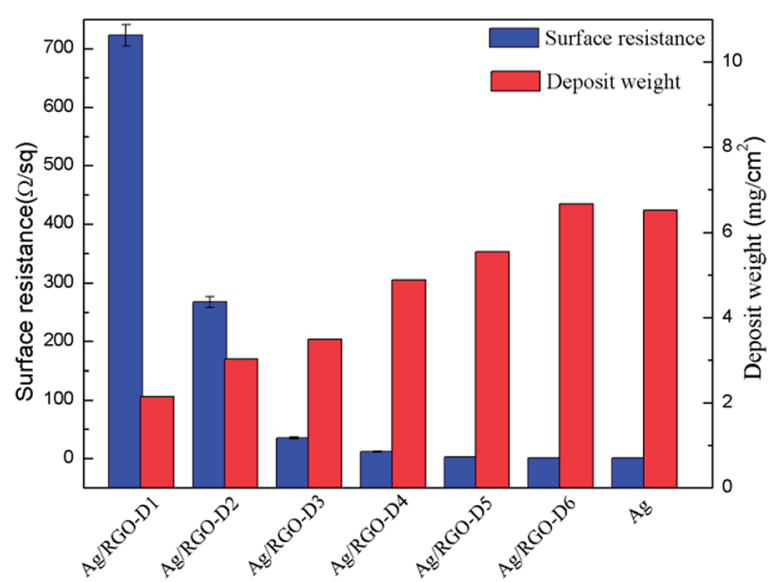

Fig. 7 Surface resistance and deposit weight of original fabric and dopamine, RGO, Ag and Ag/RGO coated PET fabrics. 


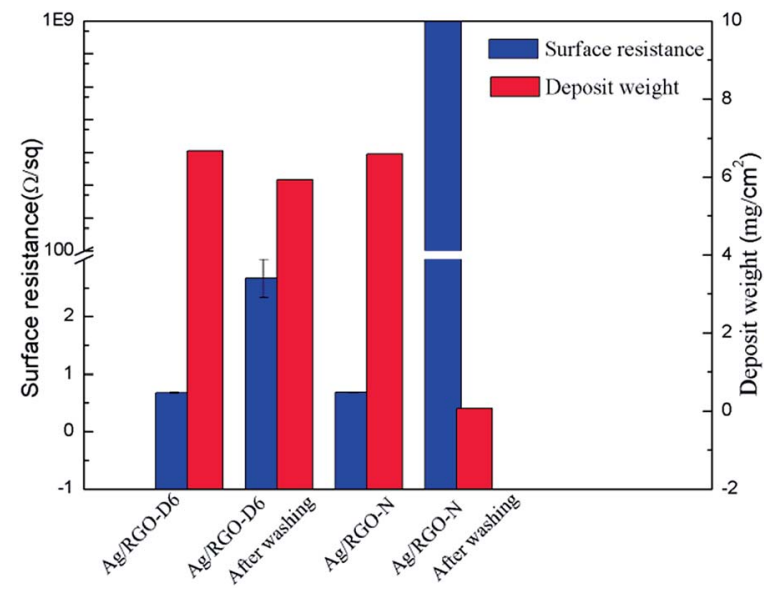

Fig. 8 Deposit weight and surface resistance of Ag/RGO-N and Ag/RGO-D6 before and after washing.

conductivity. In addition, RGO coated PET fabric also shows poor electrical conductivity because there are many functional groups in RGO including hydroxyl, epoxy and carboxyl and the structure of $\mathrm{C}-\mathrm{C}$ is incomplete, which causes defects and significantly restricts its electrical conductivity. Surface resistance of Ag coated PET fabric and Ag/RGO-D6 are $0.759 \Omega \mathrm{sq}^{-1}$ and $0.678 \Omega \mathrm{sq}^{-1}$, respectively. Electrical conductivity of Ag/RGO coated PET fabric is higher than previous report. ${ }^{16}$ The result indicates that Ag coated PET fabric and Ag/RGO-D show excellent electrical conductivity. However, electrical conductivity of $\mathrm{Ag} / \mathrm{RGO}-\mathrm{D6}$ is superior to that of Ag coated PET fabric. The phenomenon can be explained by the fact that the RGO sheets are beneficial for deposition of silver particles on the PET fabric. In addition, surface resistance of the Ag/RGO coated fabrics decreases with the rise of concentration of silver nitrate in the solution. More deposit weight results in lower surface resistance. In this study, deposit weight of coating on the fibers increases with the increase of concentration of silver nitrate, thus surface resistance of the Ag/RGO coated fabrics decreases with the rise of concentration of silver nitrate.

Surface resistance and deposit weight of $\mathrm{Ag} / \mathrm{RGO}-\mathrm{N}$ and $\mathrm{Ag} /$ RGO-D6 before and after washing are shown in Fig. 8. Ag/ RGO-N after washing shows no electrical conductivity because the deposits peels from the fabric resulted from weak Van der Waals' force between Ag/RGO coating and the fabric. In addition, surface resistance of Ag/RGO-D6 after washing is $2 \Omega \mathrm{sq}^{-1}$, which is slightly higher than that before washing. It is can be explained by that there are still lots of $\mathrm{Ag} / \mathrm{RGO}$ coating on the fibers due to coordinate bonds and electrostatic adsorption between the coatings and fibers. The result indicates that the Ag/RGO coated PET fabrics with dopamine possess excellent electrical conductivity and washing fastness.

\subsection{EMI SE of Ag/RGO coated PET fabrics}

Fig. 9 presents EMI SE of original fabric and dopamine, RGO, Ag and Ag/RGO coated PET fabrics. It is obvious that EMI SE of original fabric, dopamine and RGO coated PET fabrics are so low. The phenomenon can be explained by the fact that EMI SE

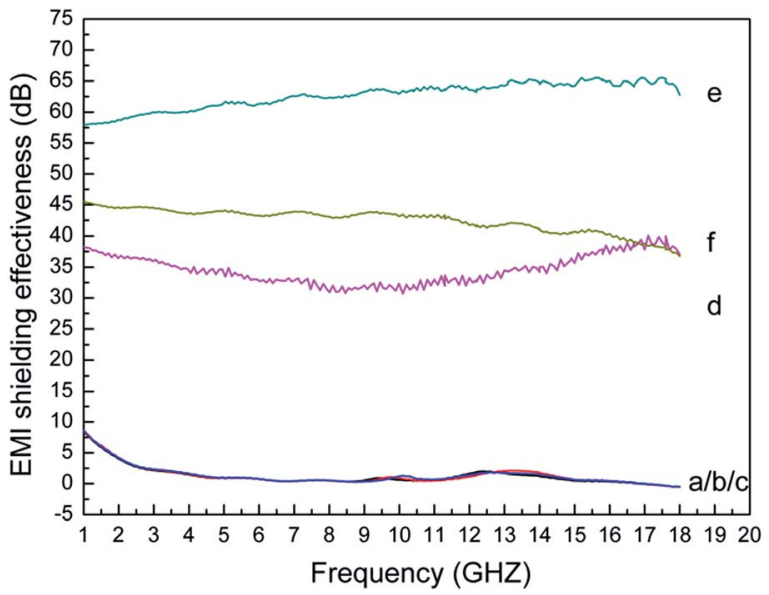

Fig. 9 EMI SE of (a) original fabric and (b) dopamine, (c) RGO, (d) Ag coated PET fabrics and Ag/RGO-D6 (e) before and (f) after washing at the frequency ranging from $1 \mathrm{GHz}$ to $18 \mathrm{GHz}$.

depends on electrical conductivity of shielding material. The electrical conductivity is a key factor to EMI SE. Accordingly, poor electrical conductivity results in low EMI SE. Obviously, EMI SE of Ag/RGO coated PET fabric ranges from $58 \mathrm{~dB}$ to $65 \mathrm{~dB}$ at the frequencies from $1 \mathrm{GHz}$ to $18 \mathrm{GHz}$, which is much higher than that coated with Ag or RGO due to the synergistic effects of $\mathrm{Ag}$ and RGO. In addition, EMI SE of Ag/RGO coated PET fabric after washing ranges from $40 \mathrm{~dB}$ to $45 \mathrm{~dB}$, which is lower than that before washing due to an increase of surface resistance after washing. Nonetheless, EMI SE of Ag/RGO coated PET fabric after washing is still more than that of $\mathrm{Ag}$ coated PET fabric.

Fig. 10 shows that EMI SE of Ag/RGO-D1, Ag/RGO-D2, Ag/ RGO-D3, Ag/RGO-D4, Ag/RGO-D5 and Ag/RGO-D6. It can be seen from Fig. 10 that EMI SE of Ag/RGO coated PET fabrics increases with rise of silver nitrate concentration. EMI SE of Ag/ RGO coated PET fabrics increases from $6 \mathrm{~dB}$ to $65 \mathrm{~dB}$ at frequencies ranging from $1 \mathrm{GHz}$ to $18 \mathrm{GHz}$ when silver nitrate

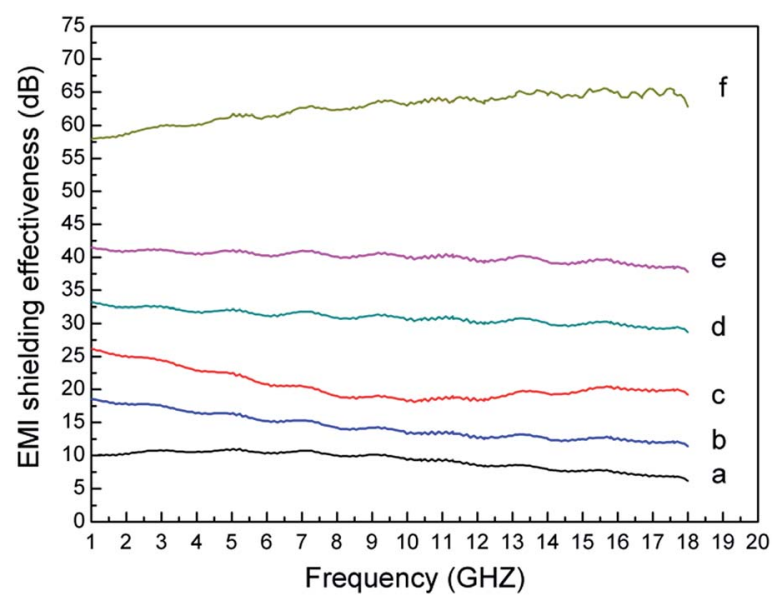

Fig. 10 EMI SE of (a) Ag/RGO-D1, (b) Ag/RGO-D2, (c) Ag/RGO-D3, (d) $\mathrm{Ag} / \mathrm{RGO}-\mathrm{D} 4$, (e) Ag/RGO-D5 and (f) Ag/RGO-D6 at the frequency ranging from $1 \mathrm{GHz}$ to $18 \mathrm{GHz}$. 
concentration increases from $1 \mathrm{~g} \mathrm{~L}^{-1}$ to $6 \mathrm{~g} \mathrm{~L}^{-1}$. The phenomenon can be explained by that better electrical conductivity results in excellent EMI SE of Ag/RGO coated PET fabrics. EMI $\mathrm{SE}$ of Ag/RGO coated PET fabric can reach $65 \mathrm{~dB}$. Until now, it has been reported about EMI shielding textile materials through pure metal or carbon material deposition on textile. ${ }^{28-30}$ Compared with previous studies, it is affirmed that $\mathrm{Ag} / \mathrm{RGO}$ coated PET fabric possesses high EMI SE property.

As is well known, EMI shielding efficiency is the combination of the reflection, absorption and multiple internal reflections of electromagnetic radiation. In previous reports, carbon materials exhibit the efficient absorption of electromagnetic energy, which is ideal absorbers. ${ }^{31-34}$ Generally, metal materials usually show the reflection of electromagnetic energy. In this study, there are a lot of silver particles deposited on the surface of RGO sheets as shown in Fig. 4. The Ag/RGO coated PET fabric probably exhibit reflection-dominated EMI shielding property. Therefore, there is a potential application of Ag/RGO coated PET fabric modified with dopamine as lightweight and flexible EMI shielding materials.

\section{Conclusions}

PET fabric is successfully modified with dopamine, and then RGO sheets and silver particles are compactly coated on the dopamine modified PET fabric under microwave irradiation. Surface resistance of Ag/RGO coated PET fabrics decreases and EMI SE increases with the rise of concentration of silver nitrate in the solution. Moreover, electrical conductivity and EMI SE of Ag/RGO coated PET fabric modified with dopamine after washing are slightly lower than that before washing. However, electrical conductivity and EMI SE of Ag/RGO coated PET fabrics with dopamine is much better than that without dopamine. The result suggests that dopamine modification on PET fabric improves adhesive strength between coatings and PET fabric. Therefore, there is a potential application of $\mathrm{Ag} / \mathrm{RGO}$ coated PET fabric modified with dopamine as lightweight and flexible EMI shielding materials.

\section{Conflicts of interest}

There are no conflicts to declare.

\section{Acknowledgements}

This work was financially supported by The National Natural Science Foundation of China (No. 51203099) and Chengdu Science and Technology Bureau (No. 2015-HM01-00380-SF).

\section{References}

1 X. C. Luo and D. D. L. Chung, Composites, Part B, 1999, 30, 227-231.

2 M. D. Stoller, S. Park, Y. W. Zhu, J. An and R. S. Ruoff, Nano Lett., 2008, 8, 3498-3502.

3 Y. A. Samad, Y. Li, S. M. Alhassan and K. Liao, RSC Adv., 2014, 4, 16935-16938.
4 A. K. Geim, Science, 2009, 324, 1530-1534.

5 C. Lee, X. Wei, J. W. Kysar and J. Hong, Science, 2008, 321, 385-388.

6 M. S. Cao, X. X. Wang, W. Q. Cao and J. Yuan, J. Mater. Chem. C, 2015, 3, 6589-6599.

7 M. Q. Cao, X. X. Wang, J. Yuan, W. Z. Wang and M. S. Cao, J. Mater. Chem. C, 2015, 3, 10017-10022.

8 B. Wen, M. S. Cao, M. M. Lu, W. Q. Cao, H. L. Shi, J. Liu, X. X. Wang, H. B. Jin, X. Y. Fang, W. Z. Wang and J. Yuan, Adv. Mater., 2014, 26, 3484-3489.

9 J. Z. He, X. X. Wang, Y. L. Zhang and M. S. Cao, J. Mater. Chem. C, 2016, 4, 7130-7140.

10 S. J. Wang, Y. W. Zhang, H. L. Ma, Q. L. Zhang, W. G. Xu, J. Peng, J. Q. Li, Z. Z. Yu and M. L. Zhai, Carbon, 2013, 55, 245-252.

11 G. Liu, Y. J. Wang, X. J. Pu, Y. Jiang, L. L. Cheng and Z. Jiao, Appl. Surf. Sci., 2015, 349, 570-575.

12 N. Sabetzadeh, S. S. Najar and S. H. Bahrami, J. Appl. Polym. Sci., 2013, 130, 3009-3017.

13 F. Z. Engin and I. Usta, Text. Res. J., 2014, 84, 903-912.

14 K. K. Gupta, S. M. Abbas and A. C. Abhyankar, J. Electromagn. Waves Appl., 2015, 29, 1454-1472.

15 V. Babaahmadi, M. Montazer and W. Gao, Carbon, 2017, 118, 443-451.

16 A. Moazami, M. Montazer and M. K. Dolatabadi, Fibers Polym., 2016, 17, 1359-1370.

17 D. P. Das, R. K. Barik, J. Das, P. Mohapatra and K. M. Parida, RSC Adv., 2012, 2, 7377-7379.

18 T. Long, L. Hu, H. X. Dai and X. Y. Tang, Appl. Phys. A, 2014, 116, 25-32.

19 A. Moradi Golsheikh, N. M. Huang, H. N. Lim and R. Zakaria, RSC Adv., 2014, 4, 36401-36411.

20 R. H. Guo, Y. N. Li, J. W. Lan, S. X. Jiang, T. Liu and W. Q. Yan, J. Appl. Polym. Sci., 2013, 130, 38623868.

21 V. H. T. Thi and B. K. Lee, J. Photochem. Photobiol., A, 2017, 338, 13-22.

22 W. C. Wang, W. J. Cheng, M. Tian, H. Zou, L. Li and L. Q. Zhang, Electrochim. Acta, 2016, 79, 37-45.

23 R. K. Wang, C. H. Shin, S. Park, L. Z. Cui, D. Kim, J. S. Park and M. Ryu, Food Chem., 2015, 226, 2241.

24 R. Sa, Y. Yan, Z. Wei, L. Zhang, W. Wang and M. Tian, ACS Appl. Mater. Interfaces, 2014, 6, 21730-21738.

25 L. H. Peng, R. H. Guo, J. W. Lan, S. X. Jiang, Y. F. He and X. M. Huang, Appl. Surf. Sci., 2016, 386, 151-159.

26 C. Xu, M. Tian, L. Liu, H. Zou, L. Zhang and W. Wang, J. Electrochem. Soc., 2012, 159, D217-D224.

27 L. Z. Li, M. X. Chen, G. B. Huang, N. A. Yang, L. Zhang, H. Wang, Y. Liu, W. Wang and J. P. Gao, J. Power Sources, 2014, 263, 13-21.

28 Y. X. Lu, S. H. Jiang and Y. M. Huang, Surf. Coat. Technol., 2010, 204, 2829-2833.

29 R. R. Bonaldi, E. Siores and T. Shah, Synth. Met., 2014, 187, 1-8.

30 L. H. Zou, C. T. Lan, X. P. Li, S. L. Zhang, Y. P. Qiu and Y. Ma, Fibers Polym., 2015, 16, 2158-2164. 
31 B. Wen, M. S. Cao, Z. L. Hou, W. L. Song, L. Zhang, M. M. Lu, H. B. Jin, X. Y. Fang, W. Z. Wang and J. Yuan, Carbon, 2013, 65, 124-139.

32 J. J. Liang, Y. Wang, Y. Huang, Y. F. Ma, Z. F. Liu, J. M. Cai, C. D. Zhang, H. J. Gao and Y. S. Chen, Carbon, 2009, 47, 922-925.
33 M. S. Cao, W. L. Song, Z. L. Hou, B. Wen and J. Yuan, Carbon, 2010, 48, 788-796.

34 Z. F. Liu, G. Bai, Y. Huang, Y. F. Ma, F. Du, F. F. Li, T. Y. Guo and Y. S. Chen, Carbon, 2007, 45, 821-827. 